
allemande

46-1 | 2014

Les fondements normatifs de l'État constitutionnel moderne en Allemagne. Une approche pluridisciplinaire

\title{
Zwischen Brücke und Bumerang
}

Was von der Bundestagswahl 2013 im Internet haften bleibt

Julia Kiegeland, Stephan Klecha et Christopher Schmitz

\section{OpenEdition}

\section{Journals}

Édition électronique

URL : https://journals.openedition.org/allemagne/1430

DOI : 10.4000/allemagne. 1430

ISSN : 2605-7913

Éditeur

Société d'études allemandes

Édition imprimée

Date de publication : 30 juin 2014

Pagination : 215-230

ISSN : 0035-0974

Référence électronique

Julia Kiegeland, Stephan Klecha und Christopher Schmitz, „Zwischen Brücke und Bumerang“, Revue d'Allemagne et des pays de langue allemande [Online], 46-1 | 2014, Online erschienen am: 29 Juli 2019 abgerufen am 21 Mai 2021. URL: http://journals.openedition.org/allemagne/1430 ; DOl: https:// doi.org/10.4000/allemagne. 1430 


\title{
Zwischen Brücke und Bumerang Was von der Bundestagswahl 2013 im Internet haften bleibt
}

\author{
- Julia Kiegeland*, Stephan Klecha**, Christopher Schmitz ${ }^{* * * *}$
}

Das Internet wird gemeinhin als Medium der Zukunft gefeiert. „Politik im Internet ${ }^{\varsigma}$ hat sich binnen eines Jahrzehnts von einer vagen Utopie zu einer Routineangelegenheit entwickelt, die kaum ein Akteur der Berufspolitik und Zivilgesellschaft ignorieren

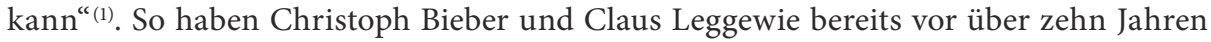
bilanziert - und man ist geneigt, ihre Einschätzung von damals fast für eine Untertreibung zu halten. Mobile Endgeräte sowie der Ausbau der Breitbandverkabelung auf der einen Seite und die wachsende Verbreitung von niedrigschwellig zugänglichen Kommunikationsplattformen auf der anderen haben die Zahl der Internetnutzer steigen lassen. Seit seiner Gründung im Jahr 2004 hat das größte Soziale Netzwerk der Welt - Facebook - etwa eine Milliarde Nutzerinnen und Nutzer angezogen. Das Internet avanciert damit immer mehr zu einem etablierten Massenmedium. Zwar nivellieren sich Nutzungsgefälle entlang von Kategorien wie Alter oder Bildungsgrad zunehmend $^{(2)}$, sind aber nach wie vor existent ${ }^{(3)}$. So sind laut einer Studie aus dem Jahr 2012 knapp 53,4 Mio Deutsche online, die allgemeine Abdeckung liegt demnach bei gut $76 \%$ der Bevölkerung. Was zunächst nach soliden Werten klingt, erfährt dadurch eine Relativierung, dass knapp 40\% der Nutzerinnen das Netz nur gelegentlich und selektiv

\footnotetext{
* Studentin im Master Politikwissenschaft an der Universität Göttingen, studentische Hilfskraft am Göttinger Institut für Demokratieforschung, Redaktionsmitglied der Zeitschrift INDES. Zeitschrift für Politik und Gesellschaft.

** Wissenschaftlicher Mitarbeiter am Göttinger Institut für Demokratieforschung.

*:* Student im Master Politikwissenschaft an der Universität Göttingen, studentische Hilfskraft am Göttinger Institut für Demokratieforschung.

1 Vgl. Claus Leggewie, Christoph Bieber, „Interaktive Demokratie. Politische Online-Kommunikation und digitale Politikprozesse", Aus Politik und Zeitgeschichte, 41-42 (2001), S. 37-45, hier S. 37.

2 Vgl. Martin Емmer u.a., Bürger online. Die Entwicklung der politischen Online-Kommunikation in Deutschland, Bonn, bpb, 2011, S. 113.

3 Vgl. Stephan EIseL, Internet und Demokratie, Freiburg, Herder, 2011, S. 164f.
} 
nutzen ${ }^{(4)}$. Gleichzeitig büßen Tageszeitung und Fernsehen keineswegs ihre angestammte Rolle bei der Verbreitung politischer Informationen ein ${ }^{(5)}$. Zumindest das Fernsehen dürfte seinen Stellenwert auch über den Generationenwechsel hinweg behaupten: Selbst bei den „Digital Natives“, der Altersgruppe zwischen 18 und 29 Jahren, verwies es das Internet 2009 auf den zweiten Rang. Und auch die vermeintlich abgehängten analogen Medien verlieren insofern nicht zwingend an Einfluss, weil sie, die Webpräsenzen der traditionellen Medien, eben Zeitungen und Zeitschriften, wiederum ganz oben auf der Liste der Informationsbeschaffungsorte im Internet stehen. Die Auftritte von Zeitungen, Zeitschriften (46\%), Radiosendern (6\%) und Fernsehanstalten (10\%) sind in drei Vierteln der Fälle die erste Wahl. Blogs, Foren (6\%), soziale Netzwerke (9\%) und sonstige Internetpräsenzen einschließlich Videoplattformen wie YouTube (15\%) kommen hingegen nur auf Zustimmungswerte von knapp einem Viertel ${ }^{(6)}$.

\section{Wahlkämpfe, Internet und Soziale Medien}

Dennoch: Die allgemeinen Entwicklungstrends lassen eine weitere Bedeutungszunahme des Mediums Internet erwarten, gerade für den Wahlkampf. Der Trend geht dabei zu einer angeblichen Anpassung der im Internet genutzten Methoden, Taktiken und Inhalte an die bewährten Offline-Kampagnen. Dies wäre insofern wenig überraschend, als sich der Wahlkampf immer weiter professionalisiert und streng an den Kriterien der Markt- und Meinungsforschung ausrichtet. Die über Jahre gewonnenen Erkenntnisse werden durch die bloße Existenz eines weiteren Verbreitungsweg ja nicht über Nacht außer Kraft gesetzt, zumal die Mehrzahl der Nutzer das Internet eben in einer eher klassischen Weise nutzt ${ }^{(7)}$. Dennoch lässt sich nicht leugnen, dass neue Verbreitungsformen stets dazu geführt haben, die Ästhetik der Wähleransprache zu verändern und das Kommunikationsverhalten an die Spezifika des jeweiligen Mediums anzupassen. Gleichzeitig ließen sich so beständig Wählergruppen neu erschließen. Deswegen wird dem Internet ein massives Potential zugetraut, das es auch bereits unter Beweis gestellt hat. Die erfolgreichen Obama-Wahlkämpfe oder die Entstehung der Piratenpartei könnte man hier - oberflächlich diagnostiziert - sicherlich als Beleg

4 Vgl. Birgit Van Eimeren, Beate Frees, „76 Prozent der Deutschen online - neue Nutzungssituation durch mobile Endgeräte“, Media Perspektiven, 7-8 (2012), S. 362-379.

5 Vgl. Martin Emmer, Jens Wolling, „Online-Kommunikation und politische Öffentlichkeit“, in: Wolfgang Schweiger, Klaus Beck (Hg.), Handbuch Online-Kommunikation, Wiesbaden, VS Verlag für Sozialwissenschaften, 2010, S. 36-72, hier S. 99ff.

6 Die Werte beziehen sich auf eine Teilfrage der Erhebung, bei der Mehrfachnennungen möglich waren. Vgl. Stefan GeEse u.a., „Berichterstattung zur Bundestagswahl 2009 aus Sicht der Zuschauer. Ergebnisse einer Repräsentativbefragung der AGF/GfK Fernsehforschung“, Media Perspektiven, 12 (2009), S. 637-650.

7 Vgl. Christina Holtz-Bacha, „Massenmedien und Wahlen: Die Professionalisierung der Kampagnen“, Aus Politik und Zeitgeschichte, 15-16 (2002), S. 23-28; Christina Holtz-BACHA, „Wahljahr 2009 - Professionalisierung verzögert?“, in: Dies. (Hg.), Die Massenmedien im Wahlkampf. Das Wahljahr 2009, Wiesbaden, VS Verlag für Sozialwissenschaften, 2010, S. 7-21, hier S. 17ff; Andreas JungherR, Harald Schoen, Das Internet in Wahlkämpfen. Konzepte, Wirkungen und Kampagnenfunktion, Wiesbaden, VS Verlag für Sozialwissenschaften, 2013, S. 125; Eva Johanna Schweitzer, „Normalisierung 2.0. Die Online-Wahlkämpfe deutscher Parteien zu den Bundestagswahlen 2002-2009“, in: Ch. Holtz-BAcha (Hg.), Die Massenmedien im Wahlkampf, a.a.O., S. $193 f$. 
anführen. Das Internet gilt darüber hinaus als eine Art Seismograph für gesellschaftliche Wandlungsprozesse und schwelende Konflikte.

Um die Dimensionen zu verstehen, mit denen und in denen das Internet einen Einfluss auf den Bundestagswahlkampf 2013 ausgeübt haben könnte, ist es zunächst notwendig, das Internet selbst ein wenig besser zu verstehen. So ist das demokratische oder wahlkämpferische Potential einerseits nicht zu erfassen, wenn andererseits nicht zuvor das technische Potential, darunter maßgeblich die Kommunikationsvorteile des Internets gegenüber anderen Medien, deutlich geworden ist. Grundsätzlich ermöglicht das Internet dem Nutzer - unter Umgehung von Informationsfiltern - Zugang zu Daten zu erlangen. Dabei kann der Nutzer Daten seinerseits zu Informationen aufbereiten und dadurch unmittelbar Einfluss auf die Interpretation von Daten nehmen. Das Internet ist also interaktiv, verknüpft dabei mit Bild, Text und Ton mehrere Sinnesebenen und gewährt obendrein eine Echtzeitkommunikation ${ }^{(8)}$. Dennoch ist das Netz dadurch anderen Kommunikationsmedien nicht zwingend überlegen, vielmehr fungiert es häufig als Medium nur für Anlässe, die ihrerseits analog Verbreitung finden, weshalb die Fokussierung auf einen reinen Online-Wahlkampf ohne gleichzeitige Offline-Kampagne wohl zum Scheitern verurteilt ist.

Insbesondere der digitale Stimmenfang gestaltet sich kompliziert. Diese Annahme speist sich aus zwei wesentlichen Kriterien: Zunächst begrenzt die funktionale Struktur der Social-Media-Communitys die potentiell ansprechbaren Personen weit mehr, als zu vermuten ist. Abgesehen davon, dass nicht jeder Bürger mit dem Internet vertraut ist, ist nur eine spezifische Teilgruppe der Gesamtwähler in den Sozialen Netzwerken derart aktiv, dass sie täglich die digitalen News erhalten und wahrnehmen. Wenngleich die Nutzungshäufigkeit des Internets weiter zunimmt, rufen laut Katrin Busemann 2013 lediglich 60\% der Internetnutzer ihr bevorzugtes Community-Profil täglich auf. Es überwiegt mit 87\% deutlich der Anteil der User, die sich nur einmal wöchentlich in ihr Social-Media-Profil einloggen ${ }^{(9)}$. Somit erreichen tägliche Posts selten das gesamte Freundes- oder Follower-Ensemble. Weiterhin unterliegt die Kommunikation mit Hilfe von Social-Media-Communitys immer einer potentiellen Selbstreferentialität. Werden mittels Partei- und Abgeordneten-Accounts neue Posts und Tweets veröffentlicht, so erreichen diese auf der ersten Kontaktebene vorrangig die Abonnenten und damit die eigenen Anhänger ${ }^{(10)}$. Potentielle neue Fans und Follower sind erst über die zweite und die folgenden Ebenen erreichbar. Dies gilt jedoch nur, wenn die bereits bestehenden Abonnenten die jeweiligen Post und Beiträge auch teilen und dadurch relevante Multiplikatoren innerhalb ihrer eigenen Freunde und Follower erreichen $^{(11)}$. Verbreitet diese tieferliegende Ebene ebenfalls die Posts und Beiträge, so

8 Vgl. Winand Gellner, Gerd Strohmeier, „Cyber-Kampagnen“, in: Andreas Dörner, Ludgera Vogt (Hg.), Wahl-Kämpfe. Betrachtungen über ein demokratisches Ritual, Frankfurt am Main, Suhrkamp, 2002, S. 164-186, hier S. 166.

9 Die Zahlen der Studie beziehen sich hierbei auf Nutzer in Deutschland ab 14 Jahren. Vgl. Katrin BuSEMANN, „Wer nutzt was im Social Web?“, Media Perspektiven, 7-8 (2013), S. 391-399, hier S. 394.

10 Vgl. Eva Johanna Schweitzer, Steffen Albrecht, „Das Internet im Wahlkampf: Eine Einführung“, in: Dies. (Hg.), Das Internet im Wahlkampf. Analysen zur Bundestagswahl 2009, Wiesbaden, VS Verlag für Sozialwissenschaften, 2011, S. 9-65, hier S. 41ff.

11 Jungherr/Schoen, Das Internet in Wahlkämpfen (Anm. 7), S. 132ff.; Christoph Neuberger u.a., Social Media im Bundestagswahlkampf 2013, Studie in Kooperation mit der Konrad-Adenauer-Stiftung 
steigt die Wahrscheinlichkeit, überhaupt das Interesse eines bisher unbekannten Users zu wecken. Der Informationsfluss erfolgt demnach ebenenweise, schubhaft, asymmetrisch und sehr willkürlich. Ein Teilen und Twittern kann zwar erbeten, jedoch nicht erzwungen werden. Nicht alle Freunde und Follower einer Parteien- oder Abgeordnetenseite teilen, liken oder twittern intensiv Beiträge, Informationen und Posts; und erst recht nicht alle erreichen dadurch andere Personen als diejenigen, die sowieso schon informiert sind. Die höchste Aktivität in den Netzwerken geht von einzelnen Usern aus, die zum einen täglich online und zum anderen bereit und willig sind, sich stetig und mit hoher Intensität im digitalen Austausch zu engagieren. Ihre Reichweite ist dabei jedoch determiniert durch die Anzahl ihrer Freunde und Follower.

Die Bemühung der Parteien und Abgeordneten, die eigene Position im Web strategisch zu festigen und politische Inhalte breit zu kommunizieren, ist nur auf der ersten Ebene partiell kontrollierbar. Innerhalb dieser Ebene werden die eigenen Mitglieder direkt angesprochen, darüber hinaus wird der Gemeinschaft ein Binnenkommunikationsraum geschaffen, der kollektiven Austausch und stetige Kommunikation ermöglicht. Die Online-Wahlkampfkommunikation kann diese Gegebenheit positiv nutzen, indem die räumlich etablierte Struktur ins Internet verlagert wird und somit raumlos vonstattengeht. Ressourcen werden gebündelt, die eigenen Reihen werden - auch ideologisch - geschlossen und ausgefüllt ${ }^{(12)}$. Zudem besteht die Möglichkeit, passive Mitglieder erneut zu motivieren und ihr Engagement zu reaktivieren.

Über Social-Media-Communitys zielgruppenspezifisch neue Sympathisanten anzusprechen, gestaltet sich in der Praxis weitaus komplizierter, eben weil sich die Selbstreferentialität dieser Social-Media-Communitys schwerlich durchbrechen lässt. Um potentiell neue Wählerstimmen zu akquirieren, bleiben daher klassische Massenmedien zum Zwecke konventioneller Wähleransprache mittels Wahlkampfstände und Wahlplakate unerlässlich. Zugespitzt ließe sich daher fragen, ob Social-Media-Communitys überhaupt geeignet sind, um dort Stimmenfang zu betreiben.

Das Problem stellt die schwer zu generierende digitale Aufmerksamkeit dar. Die Partei oder der Abgeordnete muss zunächst bewerkstelligen, potentielle Wähler kommunikativ im „Informationsrauschen“(13) des WorldWideWebs zu erreichen. Gleich einem Wahlstand in der Fußgängerzone, muss der potentielle Sympathisant zum Anhalten/ Verweilen auf dem eigenen Account gebracht werden. Folgt ein User so einem populären Beitrag, landet er auf dem Partei- oder Abgeordneten-Account, über den dann schließlich erst die politisch relevanten Inhalte zur Verfügung stehen. Anders formuliert: Die Parteien können zwar prinzipiell Wähler erreichen, zu denen sie mit ihren gewohnten Formen der Ansprache keinen Zugang mehr finden, sie stehen dort aber praktisch vor den gleichen Problemen der Rezeption ihrer Botschaften wie in der analogen Welt. Indes wäre es fahrlässig, wenn sie die damit vorhandenen Potentiale ignorieren würden, zumal ja die Hoffnung auf Substitution von Kommunikationsschwächen im analogen Bereich

und dem Vodafone Institut für Gesellschaft und Kommunikation, München/Münster, 2013, S. 117.

12 Vgl. Julia Kiegeland, Christopher Schmitz, „Quo vadis, Internet?“, politik-digital.de, 2013, http:// politik-digital.de/quo-vadis-internet/ (eingesehen 24.10.2013).

13 Vgl. Andreas Jungherr, „Twitternde Politiker: Zwischen buntem Rauschen und Bürgernähe 2.0“, in: Christoph Bieber u.a. (Hg.), Soziale Netze in der digitalen Welt. Das Internet zwischen egalitärer Teilhabe und ökonomischer Macht, Frankfurt am Main, Campus Verlag, 2009, S. 99-127, hier S. 99. 
besteht: „Das Internet wird daher auch als Kernelement der postmodernen Wahlkampfführung charakterisiert, mit dem Parteien und Kandidaten [...] auf die Unwägbarkeiten und Herausforderungen des heutigen Wählermarktes zu reagieren suchen“(14).

Weil sich aber die Internetkommunikation wiederum von der analogen Kommunikation abhebt, muss sie eine spezifische Ästhetik finden, um Resonanz zu erzielen. In seiner Untersuchung über die Erfolgsfaktoren von politischen YouTube-Videos während des Bundestagswahlkampfes 2009 hat Marko Bachl gezeigt, dass neben den klassischen Wahlwerbespots der Parteien - die auch im Fernsehen oder Kino gezeigt werden und somit nur eine Zweitverwertung darstellen - gerade jene Videos die höchste Reichweite erzielen konnten, die einen humoristischen Fokus oder einen humorvollen Inhalt hatten. „Daraus lässt sich schließen, dass Humor gerade für die exklusiven Online-Videos ein wichtiger Faktor sein kann, der zu einer größeren Verbreitung führt - wahrscheinlich getrieben durch die Motivation der Nutzerinnen und Nutzer, lustige Videos weiterzuempfehlen“(15). Dieser Befund lässt sich von Videos mühelos auf andere Inhalte und Darstellungsformen in den Social-Media-Communitys übertragen: So wurden während des Bundestagswahlkampfs über Twitter beispielsweise überwiegend die Kurznachrichten von Comedians verbreitet („retweetet“), die eine pointierte, humoristische und emotionalisierende Wertung eines Sachverhalts kundtaten ${ }^{(16)}$. Die Verwendung von authentischem Witz oder Ironie erhöht die Chance, dass Posts und Tweets binnen kürzester Zeit eine enorme Aufmerksamkeit erregen und darüber wiederum von den klassischen, analogen Medien selbst aufgegriffen und weiterverbreitet werden. Umgekehrt werden Geschehnisse, die analog verbreitet werden, im Internet oft in ironischer, sarkastischer oder zynischer Form weiterverarbeitet. Die Trennung zwischen der Internetgemeinde und dem analogen Kommunikationsraum ist dadurch fließend.

Im Folgenden wird anhand verschiedener Beispiele verdeutlicht, wie gerade Ironie die Funktionsweise von wahlkampfpolitischen Inhalten im Internet, vorzugsweise in den Social-Media-Communitys, beeinflussen, strukturieren, beflügeln, aber auch behindern und einschränken kann. Hierbei bietet sich eine kategoriale Unterscheidung zwischen zwei Typen der ironischen Wirkungsweise an. Zum einen handelt es sich um die "genuine Ironie“: Diese liegt vor, wenn der ironische Inhalt bereits bewusst Teil der politischen Botschaft ist und deshalb ohne Verfremdung weiter verbreitet oder popularisiert wird. Zum anderen findet sich das, was wir als „verkehrte Ironie“ bezeichnen wollen: Die authentische und wahrhaftige politische Kommunikation wird so rezipiert, dass die eigentliche Aussage verdreht und dadurch ironisiert wird. Da die momentane Funktionsweise des Internets dazu tendiert, die klassische Dichotomie zwischen Binnen- und Außenkommunikation ${ }^{(17)}$ tendenziell zu konterkarieren, ist es ratsam, die Funktionsweise von Ironie in ein Verhältnis zur Selbstreferentialität zu setzen, bevor die Beispiele zeigen, wie diese kommunikative Sackgasse durch Ironie überwunden werden kann.

14 E. J. Schweitzer, „Normalisierung 2.0.“(Anm. 7), S. 193.

15 Vgl. Marko BACHL, „Erfolgsfaktoren politischer YouTube-Videos“, in: Schweitzer/Albrecht (Hg.), Das Internet im Wahlkampf (Anm. 10), S. 171f.

16 Ch. Neuberger u.a., Social Media im Bundestagswahlkampf 2013 (Anm. 11), S. 112.

17 Leggewie/Bieber, „Interaktive Demokratie“(Anm. 1), S. 38. 


\section{Selbstreferentielle Kommunikation in der Filter Bubble}

Dadurch, dass sich die Wahlkampfkommunikation der Parteien ohnehin in hohem Maße nach Innen richtet oder von den konventionellen Medien übersehen wird, ergibt sich hier notwendigerweise ein Zustand der Selbstreferentialität, der auch im Internet seine Entsprechung findet. Wenn die Kommunikation von Parteien dort nicht über die Kreise der eigenen Klientel hinaus gelangt, droht ein sich selbst verstärkender Kreislauf der Selbstbestätigung. Informationen erreichen dabei nur jene Personen, die ohnehin mit der Partei sympathisieren oder gar Mitglied sind. Oder anders gesagt: Wer nicht ohnehin eine Präferenz für eine Partei hat - oder ihn nicht z.B. berufliche Gründe dorthin führen -, der wird keinesfalls zwingend dem Webauftritt einer Partei folgen.

Letztlich basiert jeder Gruppenzusammenschluss auf einer gewissen ideologischen Homogenität oder Interessenkonvergenz der beteiligten Individuen. Wenn sich solche Gruppen dann vom Rest der Gesellschaft abschotten, tendieren diese dazu, die Meinungen, Argumente und Weltbilder, die innerhalb der Gruppe kursieren, zu verstärken und zu radikalisieren. Der Einfluss mäßigender Meinungen auf das eigene Weltbild wird dadurch minimiert. Russel Hardin hat dieses Phänomen einmal in Hinblick auf Fanatismus und Extremismus untersucht. Dieser attestiert solchen Gruppierungen eine Tendenz zu einer verkrüppelten Epistemologie. Fanatismus ist, aus dieser Perspektive, zunächst einmal ein hauptsächlich soziologisches Phänomen ${ }^{(18)}$. Cass Sunstein nimmt diesen Ansatz auf, abstrahiert jedoch vom Themenfeld des Extremismus und spricht allgemein von Informationskokons ${ }^{(19)}$. In einem regulären politischen Kommunikationsprozess mit pluralistisch verfassten Massenmedien minimiert sich diese Gefahr, weil die Parteien beispielsweise gezwungen sind, ihre Positionen gegenüber konträren Ansichten zu verteidigen und sie im Zuge dieser Auseinandersetzung zu modifizieren und abzuschleifen.

Nun hat Eli Pariser, Geschäftsführer der amerikanischen Kampagnenwebsite MoveOn, ein solches Phänomen für das Internet unter dem Begriff der Filter Bubble (Filterblase) popularisiert ${ }^{(20)}$. Der gegenseitige Austausch von Informationen zur Generierung und Bildung geteilter Erfahrungen und allgemeiner Entscheidungsgrundlagen sei die Grundlage eines vernünftigen gesellschaftlichen Miteinanders. Die Filterblase würde diesem Trend gleich der Zentrifugalkraft entgegenwirken: „Der Grundcode des neuen Internets ist recht simpel. Die neue Generation Internetfilter schaut sich an, was Sie zu mögen scheinen - wie Sie im Netz aktiv waren oder welche Dinge oder Menschen Ihnen gefallen - und zieht entsprechende Rückschlüsse“(21). Grundlage dessen sind Cookies, auch Bugs oder Beacons genannt, die das Surfverhalten protokollieren,

18 Vgl. Russel Hardin, „The Crippled Epistemology of Extremism“, in: Albert Breton u.a. (Hg.), Political Extremism and Rationality, Cambridge, Cambridge Univ. Press, 2002, S. 3-22, hier S. 10.

19 Vgl. Cass R. Sunstein, „How Voters Can Escape From Information Cocoons“, BloombergView, 2012, http://www.bloomberg.com/news/2012-09-03/how-voters-can-escape-from-information-cocoons. html (eingesehen 04.09.2012).

20 Vgl. Eli PARISER, Filter Bubble. Wie wir im Internet entmündigt werden, München, Carl Hanser Verlag, 2012.

21 Ebd., S. 17. 
aggregieren und daraus Rückschlüsse generieren ${ }^{(22)}$, um Werbung oder Botschaften zielgerichtet senden zu können. Dieses Prinzip bringt es aber etwa bei der Nutzung von Suchmaschinen auch mit sich, dass die Eingabe desselben Suchbegriffs an unterschiedlichen Rechnern bei verschiedenen Nutzern schließlich auch unterschiedliche Suchergebnisse ergibt. Zum Problem wird das Ganze, weil die Inhalte durch einen technischen Eingriff gefiltert werden, der sich der Kenntnis der Nutzerinnen weitestgehend entzieht. „Die Maschine“, so Oliver Leistert, „ist selbst am Geschehen beteiligt, indem sie lenkt und rankt“(23).

Seitdem das Medium Internet zunehmend in den Fokus der politischen Kommunikation gerückt ist, beschreibt diese Entwicklung nicht nur einen datenschutzrechtlich relevanten Sachverhalt, sondern hat Auswirkungen auf die politische Informationssuche via Internet. Dort werden dadurch vor allem jene Medienangebote bevorzugt angesteuert, die den jeweiligen politischen Wertvorstellungen entsprechen, während Angebote mit ideologisch konträren Positionen seltener besucht werden. Inhalte und Informationen werden dadurch einseitig rezipiert. Die Social-Media-Auftritte der Parteien errichten um sich herum also tendenziell Informationskokons, die hauptsächlich Mitglieder und Sympathisanten einschließen und - abgesehen von professionellen Beobachtern - keine darüber hinausgehende Resonanz erzielen. Während es für die Bereitstellung von argumentativem und informativem Material oder organisatorischen Anliegen kein Nachteil ist, möglichst nur die eigene Klientel zu erreichen, verstärkt dieser Informationskokon die ohnehin vorhandene Reichweitenschwäche der Parteien. Die Durchdringung der Gesellschaften durch die Parteien ist - bei gewissen Abstrichen in Bezug auf die CSU - rückläufig. Das heißt, der Kreis der festen Mitglieder, Anhänger und Sympathisanten schwindet und wird obendrein älter, also tendenziell inaktiver. Für die Parteien bedeutet das, dass sie Wege finden müssen, um ihre kommunikativen Inhalte über den Kreis dieser eigenen Klientel hinaus zu verbreiten. Dabei müssen sie gerade jene Personengruppe erreichen, die der Politik im Allgemeinen oder auch der einzelnen Partei im Besonderen nicht sehr nahe steht oder sich einfach indifferent verhält. Eingedenk der Tatsache, dass die meisten Profile in den sozialen Netzwerken genutzt werden, um sich über Neuigkeiten im Freundes- und Bekanntenkreis zu informieren, statt Nachrichtenportale anzusteuern ${ }^{(24)}$, bestehen hier zwar die größten Chancen, Aufmerksamkeit zu erlangen - doch in der Praxis steht dem die Schließung der Filter Bubble entgegen.

\section{Filterblasen und Ironienadeln}

„Das Gegenteil von Ironie ist gesunder Menschenverstand“(25). Mit dieser Sentenz erfasst Richard Rorty in Kontingenz, Ironie und Solidarität den Begriff der Iro-

22 Vgl. David Berry, „Against Remediation“, in: Geert Lovink, Miriam RAsch (Hg.), Unlike Us Reader. Social Media Monopolics and their Alternatives, Amsterdam, Institute of Network Cultures, 2013, S. 31-49, hier S. 37ff.

23 Vgl. Oliver Leistert, „Der Beitrag der Social Media zur Partizipation“, Forschungsjournal Soziale Bewegungen, 2 (2013,) S. 39-48, hier S. 41.

24 K. Busemann, „Wer nutzt was im Social Web?“(Anm. 9), S. 394.

25 Vgl. Richard Rorty, Kontingenz, Ironie und Solidarität, Frankfurt am Main, Suhrkamp, 1989, S. 128. 
nie. Gesunder Menschenverstand bedeutet, dass die Umwelt und Umgebung eines Menschen in abgeschlossenen, hermetischen, in sich schlüssigen Narrativen, Weltbildern oder Begrifflichkeiten strukturiert und geordnet ist. Ironie als Gegenteil dieser geschlossenen Sichtweise zielt also auf eine Thematisierung und Sichtbarmachung von Zuständen jenseits dieses Bildes. Es geht demnach um die Thematisierung von Kontingenzen. Ironisches „Denken wird von einer ambivalenten und damit äußerst menschlichen Perspektive getragen, ist verstehend, aber nicht verabsolutierend “(26). So gesehen dient Ironie dazu, den eigenen Standpunkt als besonders reflektiert hervorzuheben. Im Zweifel lässt sich mit Hilfe von Ironie auch Kritik vorwegnehmen, indem man betont, „dass man diese Dinge doch sowieso schon bedacht hätte“(27).

Ironie kann aber auch als ein rhetorisches Stilmittel begriffen werden, welches einen elitären und distanzierten Bias eines Senders voraussetzt. Bei dieser akteurszentrierten Betrachtungsweise fordert ihr strategischer Einsatz die Kompetenz, paradoxale und dilemmatische Zusammenhänge grundlegend $\mathrm{zu}$ erkennen und diese aufzuzeigen. Dabei bezieht sich diese kritische Wahrnehmung keinesfalls ausschließlich auf andere Personen und Institutionen. Es ist durchaus möglich, dass der Sender die eigenen Tätigkeiten ironisch aufarbeitet und so sein Scheitern öffentlich thematisiert. Ironie erlaubt eine Entmystifizierung von Autoritäten, ein Exponieren von Widersprüchlichem und Abstrusem - ohne dass sich Handlungszwänge oder Veränderungsbedarf automatisch anschließen ${ }^{(28)}$. Im Gegensatz zu einem Skandal, der in den Medien in alle Richtungen ausufern kann, bietet Ironie eine tendenziell kurzweilige und zumeist gnädigere Reaktion seitens der Bevölkerung. Es wird geschmunzelt und gewitzelt, allerdings ohne weitreichende personelle oder strafrechtliche Folgen. Oder anders gewendet: Es mag zwar vorkommen, dass ein Skandal ironisiert wird; selten allerdings wird die Ironie tatsächlich skandalisiert. Auch aus diesem Grund bietet Ironie die Möglichkeit, ideologische Grenzen zu übergehen und die Gesellschaft als Ganzes anzusprechen. Ironie besitzt eine transideologische Natur des Politischen ${ }^{(29)}$.

Mit Rückblick auf die problematischen „Schranken“in den Social Media Communitys bietet die Ironie eine methodische Perspektive, um über eine Filter Bubble hinaus eine breite Wählergruppe anzusprechen. Ironie ähnelt in ihrer Funktionsweise dabei einem gut sichtbaren Wahlplakat an einem öffentlichen Platz. Ironie kann eine kommunikative Methode darstellen, die einen breiten Aufmerksamkeitsmoment seitens der Rezipienten für die eigene Botschaft generiert, welcher im Online-Wahlkampf durchaus strategisch nutzbar ist. Die Ironie ist die Nadel, die die Blase zum Platzen bringen kann. Warum aber, wenn Ironie doch so einen vielversprechenden „Grenzöffner“ darstellt, wird sie nicht öfters von Parteien und Abgeordneten strategisch genutzt? Es exis-

26 Vgl. Jöran Klatt, „Hannah und die Ironie. Hans Landa, ein (post)moderner Adolf Eichmann?“, INDES. Zeitschrift für Politik und Gesellschaft, 2 (2012), S. 141-147, hier S. 144.

27 Vgl. David Bebnowski, „Aus Angst wird Ironie. Wie eine tief verunsicherte Generation den humorigen Spaßbürger mimt“, INDES. Zeitschrift für Politik und Gesellschaft, 1 (2012), S. 39-45, hier S. 45.

28 Vgl. Detlef SACK, „Regieren und Ironie“, in: Björn Egner u.a. (Hg.), Regieren. Festschrift für Hubert Heinelt, Wiesbaden, VS Verlag für Sozialwissenschaften, 2012, S. 157-169, hier S. 157ff.

29 Vgl. Walter ReEse-Schä FER, „Die Tücke der Ironie und die Ehrlichkeit des Zynismus. Zur Dekonstruktion eines Vorurteils“, in: Thorsten Bonacker u.a. (Hg.), Die Ironie der Politik. Über die Konstruktion politischer Wirklichkeiten, Frankfurt am Main, Campus Verlag, 2003, S. 122-139. 
tiert durchaus ein kleiner, aber bedeutender Stolperstein: Ironie muss authentisch sein, um eine Brücke zwischen dem Binnen- und Außenkommunikationsraum zu schlagen. Ist sie hingegen die Essenz der verkehrten Botschaft, die von außen übergestülpt wird, dann fällt sie mitunter auf die Urheber zurück wie ein Bumerang.

\section{Von Pferden, Händen und Plagiaten: verkehrte Ironie}

Tatsächlich gibt es einige Beispiele aus den jüngsten Wahlkämpfen. Sie sind zwar rar gesät, haben aber doch eine beachtliche Wirkung entfaltet. In Form von Memen gedeihen sie im Internet und durchschreiten die ohnehin durchlässige Grenze zur analogen Kommunikation, so dass ein sich gegenseitig verstärkender Wahrnehmungsprozess in Gang gesetzt wird.

\section{Horses and Bayonets}

Das so genannte „Horse and Bayonet“-Mem aus dem letzten US-Präsidentschaftswahlkampf liefert dafür ein gutes Beispiel. Es hat seinen Ursprung in einer TV-Debatte zwischen dem Amtsinhaber Barack Obama und seinem republikanischen Herausforderer Mitt Romney: Letzterer erläuterte seine Kritik an der Finanzierung des USMilitärs und argumentierte, dass nach Haushaltskürzungen die US-Navy weniger Schiffe habe als im Jahre 1917, was für ihn nicht hinnehmbar sei. Obama reagierte auf diese Anschuldigung, indem er unumwunden zugab, dass das US-Militär tatsächlich weniger Schiffe habe als 1916 - genauso, wie das US-Militär heutzutage weniger Pferde und Bajonette (englisch „horses and bayonets") einsetze, weil sich die Anforderungen an das Militär geändert hätten. Stattdessen verfüge es nun über diese Dinger, die man Flugzeugträger nenne, und es besitze auch diese Schiffe, die tauchen könnten und als atomgetriebene U-Boote bekannt seien ${ }^{(30)}$.

Obama ist somit die ironische Verkehrung einer als authentisch geplanten Botschaft gelungen, indem er die Klage über die geringere Anzahl von Schiffen als Anachronismus darstellte, die veränderten Anforderungsprofile darlegte und das ganze ironisch zuspitzte. Diese Reaktion wurde im Internet dankbar aufgegriffen und weiterverwertet. Nur zehn Minuten, nachdem Obama sich derart geäußert hatte, gab es den ersten tumblr-Blog, der diese Wendung aufnahm. Noch bevor die Fernsehdebatte beendet war, hatte sich das Thema in Blogs, Foren und bei Twitter derart verbreitet, dass „horses and bayonets" der am häufigsten verwendete Suchbegriff des Abends bei Google wurde ${ }^{(31)}$. Die besondere Stärke des Internets war dabei dessen Multimedialität. So wurde bei tumblr ein Bild gehostet, auf dem sich Obama zu seinem Vize-Präsidenten Joe Biden herunterbeugt und ihn fragt: „Iran might get a nuke. Do we have enough horses and bayonets?“(32) Interessant an diesem Beispiel ist zugleich, dass in diesem Fall ohne das

30 ABC News, „Obama to Romney: U.S. Uses Less ,Horses and Bayonets` Today - Presidential Debate 2012“, YouTube, http://www.youtube.com/watch?v=-IW6PwJYcOc (eingesehen 26.10.2013).

31 Vgl. Adam Clark Estes, „,Horses and Bayonets' Goes from Obama's Mouth to Parody Tumblr in 9 Minutes“, The Atlantic Wire, http://www.theatlanticwire.com/politics/2012/10/it-took-less-30-minutes-horses-and-bayonets-become-meme/58227/ (eingesehen 26.10.2013).

32 o.V., „Horse And Bayonet. Appreciating Horses, Along With Bayonets“, tumblr.com, 2012, http:// horseandbayonet.tumblr.com/ (eingesehen 26.10.2013); o.V., „horsesandbayonets“, tumblr.com, 2012, http://horsesandbayonets.tumblr.com/ (eingesehen 26.10.2013). 
genaue Wissen um den Kontext - also dass die Aussage von Obama stammt und gegen Romney gerichtet war - der Eindruck entstehen könnte, dass hier Obama und Biden ironisiert werden. Romney kommt in dieser Variante überhaupt nicht vor. Andernorts kursierten Bildmontagen, in denen Romney zu Pferd sitzend in den Krieg zieht.

\section{Liberale oder rechtsdrehende Milchkultur?}

Parallel zu der stetig fortschreitenden Wahlkampf-Professionalisierung wächst auch der grafisch-visuelle Anspruch an politische Werbemittel wie Faltblätter, Plakate und Videos. Um ein stringentes und sinnstiftendes Erscheinungsbild zu gewährleisten, beauftragen mittlerweile fast alle Parteien kommerzielle Werbeagenturen. Ist das Budget etwas enger, greifen die Grafiker durchaus auf so genanntes Stock-Material zurück. Darunter sind Bilder, Illustrationen und Videosequenzen aus Datenbanken zu verstehen, die bei hoher Qualität preisgünstig immer wieder verkauft werden. Wenn nun diese Datenbanken allzu undifferenziert und unüberlegt benutzt werden, kann es zu folgender, im Internet aufgetanen, problematischen Mehrfachverwendung kommen: Die rechtsextreme NPD und die liberale FDP nutzten für ihre am 22 . August ${ }^{(33)}$ beziehungsweise am 26. August $2013^{(34)}$ bei YouTube online gestellten Werbespots die identische Aufnahme einer glücklichen Familie beim Fahrradfahren, um ihre eigentlich divergenten politischen Positionen visuell zu unterlegen. Zusätzlich zu dieser bereits unvorteilhaften Doublette wurde dieselbe Filmsequenz auch von einem finnischen Quarkhersteller genutzt. In den Socia-Media-Communitys popularisierte sich die Gegenüberstellung aller Aufnahmen rasant. Auf Facebook wurde das Bild bisher 20.236 mal geteilt und 6.994 mal geliked $^{(35)}$, auf Twitter verbreiteten sich zahleiche ironische Bemerkungen und selbst Zeitschriften wie die Süddeutsche Zeitung titelten „Liberal, rechtsextrem und ziemlich im Quark“(36). Die Onlineportale von Spiegel ${ }^{(37)}$, Zeit $^{(38)}$, Stern $^{(39)}$ und Bild ${ }^{(40)}$ informierten ihre Leser ebenfalls über diesen ungeplanten

33 NPD, „NPD-Werbespot zur Bundestagswahl 2013“, YouTube, http://www.youtube.com/watch?v= 6SiiBpydWbc (eingesehen 13.11.2013).

34 FDP, „FDP Wahlspot 2013“, YouTube, http://www.youtube.com/watch?v=IQQ5nREaJWs\&feature=y outu.be (eingesehen 13.11.2013).

35 So einzusehen auf der Facebook-Präsenz der Werbeagentur die wegmeister. kreativagentur, „FDP. NPD. Quark. die wegmeisters Fotos“, 2013, facebook.com, https://www.facebook.com/photo. php?fbid $=519690848113856 \&$ set $=$ pb.136370103112601. $-2207520000.1384349854 . \&$ type $=3 \&$ theater (eingesehen 13.11.2013).

36 Vgl. Michael KöNig, „Liberal, rechtsextrem und ziemlich im Quark“, Süddeutsche.de, 2013, http:// www.sueddeutsche.de/politik/wahlwerbespots-von-npd-und-fdp-liberal-rechtsextrem-und-ziemlich-im-quark-1.1756034 (eingesehen 13.11.2013).

37 Vgl. Anett Meiritz, „Der Quark der FDP“, Spiegel Online, 2013, http://www.spiegel.de/politik/ deutschland/wahlspot-mit-bruederle-fdp-nutzt-dasselbe-bildmaterial-wie-npd-a-918900.html (eingesehen 13.11.2013).

38 o.V., „FDP und NPD werben mit derselben Familie“, Zeit Online, 2013 http://www.zeit.de/politik/ deutschland/2013-08/fdp-npd-wahlspot-familie-quark (eingesehen 13.11.2013).

39 o.V., „Eine Familie für alle Fälle“, stern.de, 2013, http://www.stern.de/politik/linsengericht/spots-vonfdp-npd-und-fuer-finnischen-quark-eine-familie-fuer-alle-faelle-2053836.html (eingesehen 13.11.2013).

40 Vgl. Hanno KAutz, „Eine Familie für FDP, NPD \& Quark“, Bild.de, 2013, http://www.bild.de/politik/ inland/bundestagswahl/wahl-spot-panne-fdp-wirbt-mit-gleicher-familie-wie-npd-32005244.bild. html (eingesehen 13.11.2013). 
Gemeinschaftskauf. Die FDP reagierte und entfernte die Familie aus ihrem Spot. Doch das Netz vergisst nicht, und so behält eine Internetseite diesen Fauxpas im digitalen Gedächtnis fest ${ }^{(41)}$.

In diesem Fall konnte der potentielle digitale Binnenkommunikationsraum der beiden politischen Sender geweitet werden, selbst die klassischen Medien griffen die Werbespots thematisch auf. In der Netzgemeinde bündelte sich ein hoher Grad an Aufmerksamkeit - die eigentlichen Inhalte jedoch, die beide Parteien in ihren Wahlwerbespots zu kommunizieren beabsichtigten, gerieten, nach der ironischen Reflexion der Internetgemeinde, in den Hintergrund. Im Fokus blieb, insbesondere aufgrund der ideologischen Diskrepanz beider Parteien, die unbeabsichtigte optische Gemeinsamkeit von FDP, NPD - und Quark. Eine authentisch durchdachte und glaubwürdige Kommunikation seitens der jeweiligen Parteien wurde ad absurdum geführt. Besonders treffend kommentiert dies eine Leserin unter der Meldung auf Zeit Online: „Man denkt man ist clever und schnappt sich ein paar billige Klischeefotos, statt etwas Authentisches und Ehrliches selbst produzieren zu lassen“(42). Auch wenn nicht festzustellen ist, wie viele Aufrufe der jeweiligen Videos (das Video der NPD hat fast 430.000, das der FDP mehr als 350.000 Aufrufe) genau dieser Berichterstattung geschuldet sind: Die mediale Aufmerksamkeit war massiv. Die hier verwendete Ironie ist in dem Sinne verkehrt, dass sie grundlegend von keinem der beiden politischen Sender beabsichtigt wurde, ihre inhaltliche Botschaft durch die Ironie jedoch benachteiligt wurde.

\section{Deutschland in guten Händen}

„2378 Quadratmeter groß, zusammengesetzt aus mehr als 2000 Aufnahmen, die zu einer Raute geformten Hände von Bundeskanzlerin Angela Merkel“(43): Dieser Eindruck prägte ab Anfang September die Szenerie am Berliner Hauptbahnhof. Ein gigantisches Wahlplakat, ein Mosaik aus tausenden Fotos, eingesendet von Mitgliedern, Sympathisantinnen und Unterstützerinnen der Union, dazu der Slogan „Deutschland in guten Händen“. Das Markenzeichen der Kanzlerin, die zur Raute geformten Hände, sollen ihren politischen Stil in einer Art und Weise symbolisieren, dass es genügt, nur diese Hände zu zeigen und auf die Darstellung ihres Gesichts schlichtweg zu verzichten. "Merkels Markenzeichen ist der Postheroismus, der kein Pathos kann.“ Unideologischer Pragmatismus, Integrität durch eine Politik um der Sache willen ${ }^{(44)}$ - das ist das Prinzip Merkel. Und die zur Raute geformten Hände fassen die Eigenschaften in einer zum Markenzeichen gewordenen, authentischen Geste zusammen. Dafür sollte das Plakat stehen, verstärkt durch den Symbolismus des Mosaiks, das hier für Deutschland und seine Bürger steht. Und für die Passanten, die dieses gigantische Plakat unmittelbar sahen, als sie unter ihm vorbeischritten, mag dies in vielen Fällen auch so gewirkt haben. Vielleicht stieß es einigen sauer auf, dass thematische Politik hinter die reine Geste, das Symbol zurücktrat, doch die Authentizität dieses

41 http://fdp-npd-quark.de/ (Stand: 18.12.2013).

42 Interpunktionsfehler im Original.

43 Vgl. RalfSchönball, Henning Onken, „Merkel wird Mr. Burns nicht los“, Der Tagesspiegel, 2013, http:// www.tagesspiegel.de/berlin/umstrittenes-wahlplakat-merkel-wird-mr-burns-nicht-los/8742138.html.

44 Vgl. Katharina Trittel, „Die CDU oder: Es reicht - immer noch“, INDES. Zeitschrift für Politik und Gesellschaft, 2 (2013), S. 132-135, hier S. 133. 
etablierten Bildes mag dies überstrahlt haben. Im Internet allerdings wurde diese Interpretation an vielen Stellen nicht geteilt. Die globale, fürsorgende Geste wurde in weiten Teilen der Social-Media-Communitys dekonstruiert, durchbrochen, verkehrt, eben ironisiert. Möglich wurde dies, indem die eigentliche Stärke des Plakats, der Botschaft auch ohne die Darstellung eines Gesichts oder eines kompletten Oberkörpers Authentizität verleihen zu können, in ihr Gegenteil verkehrt wurde. Fotomontagen nahmen die Vorlage auf und ironisierten den Slogan des Plakats, die Geste der Kanzlerin, indem Oberkörper und Gesichter von anderen Figuren und Persönlichkeiten in das Bild geschnitten und auf das Plakat gesetzt wurden: Die Bandbreite der Personen reicht von Mr. Burns, dem skrupellosen Atomkraftwerkbetreiber aus der Kultserie Die Simpsons, über Darth Sidious - dem Imperator, Diktator und Lehrmeister Darth Vaders - aus der Star Wars-Saga, Dr. Evil, dem Bösewicht aus der Austin PowersFilmreihe, hin zu Chuck Norris, Alice Schwarzer, Mao oder Lenin. Das Stereotyp der Botschaft wurde umgekehrt und instrumentalisiert. Die Vorstellung, Deutschland in den ,guten Händen` eines Mao Zedong zu wissen, der auch durch die Millionen Tote während seiner Herrschaftszeit im kollektiven Gedächtnis verhaftet bleibt, führt die Aussage des Plakats völlig ad absurdum und legt die Kontingenz dieser scheinbar so eindeutigen Botschaft geschickt bloß. Hierbei handelt es sich tatsächlich um eine reine Reaktion aus den Reihen des Internets und der sozialen Netzwerke. Zwar war der Verbreitungsgrad dieser Aktion mäßig - die erste Bearbeitung erschien auf der Website 9gag.com und zeigte Merkels Hände und Mr. Burns Gesicht. Ein knappes Drittel aller Nutzerinnen findet über Facebook auf die Seite, knapp 7\% der 9gag-Besucher kommen aus Deutschland, wo diese Website im nationalen Ranking der Aufrufe den immerhin 214. Platz belegt ${ }^{(45)}$. Die potentielle Reichweite ist also hoch, und die Tatsache, dass mit dem Tagesspiegel immerhin (wenn auch nur in der Online-Ausgabe) ein konventionelles Medium dieses Phänomen aufgegriffen hat, deutet auf eine erfolgreiche Rückkopplung der ironisierten Botschaft hin, die Deutungshoheit jener Berichte, die nur über das originale Plakat berichteten, wurde zumindest in Frage gestellt.

\section{Plumpbeutler und Metalllegierungen: genuine Ironie}

\section{Ein Wombat in jedem Haushalt}

Den Slogan „Ein Wombat in jedem Haushalt. Unrealistische Wahlversprechen können wir auch!“(46) plakatierte die Piratenpartei Sachsen-Anhalt im Wahlkampf 2013 zu Beginn lediglich an zehn Laternen. In den Sozialen Netzwerken verbreitete sich das „Wombatplakat“ in elektronischer Form umso rasanter und steigerte sich in seiner Popularität dermaßen, dass es parallel auf Papier nachgedruckt wurde. Im Web wurde geklickt, geliked und geteilt. Ein eigener Hashtag entstand und zahlreiche, auch politikferne, Blogs verbreiteten das Plakat weiter. Auf ihrer Webseite und ihren Social Media Accounts bezieht die Piratenpartei ergänzend deutlich Stellung und argumentiert ihr Anliegen, Bürger überzeugen zu wollen, Wahlprogramme zu lesen, anstatt

45 http://www.alexa.com/siteinfo/9gag.com (Stand: 13.11.2013).

46 Piratenpartei, „Für einen Wombat in jedem Haushalt“, Piratenwiki, 2013, http://wiki.piratenpartei. de/Datei:Wombat_Plakat_Final.jpg (eingesehen 13.11.2013). 
Wahlplakaten blind zu vertrauen. In dieser Hinsicht gleicht der Wombat stilistisch und inhaltlich der Ideenkopierer-Kampagne des niedersächsischen Landesverbandes der Piratenpartei für die Landtagswahl im Januar 2013. Hier waren die Wahlplakate im Corporate Design verschiedener großer Firmen oder Organisationen - darunter IKEA, Apple, Deutsche Telekom, McDonald's oder auch Greenpeace - gehalten und beabsichtigten damit, zum Nachdenken über die Aufmachung und Aussagekraft von Wahlplakaten anzuregen ${ }^{(47)}$. Im Gegensatz zum Wombat-Plakat war aber die Verbindung zur Piratenpartei nicht deutlich genug erkennbar, weshalb die Kampagne zum Teil kritisch betrachtet und ihre Wirkungskraft in Frage gestellt wurde ${ }^{(48)}$.

Ironie war für die Piraten dabei kein neues Instrument im Wahlkampf. Bereits zur Abgeordnetenhauswahl in Berlin im Herbst 2011, bei denen die Piraten erstmals in ein deutsches Landesparlament einziehen konnten, wurden ironische Plakate verwendet: Eines zeigt das Gesicht des jetzigen Abgeordneten Christopher Lauer in Verbindung mit einer ironischen Aussage: „Warum häng ich hier eigentlich, ihr geht ja eh nicht wählen“"(49). Hieran wird deutlich, dass die Piratenpartei als eine Formation mit dezidiert netzkulturellen Wurzeln ${ }^{(50)}$ verstehbar ist. Über die tatsächliche und absolute Reichweite des Plakates kann nur gemutmaßt werden. Google immerhin liefert unter der Suchkombination „Wombat in jedem Haushalt Piraten“ ungefähr 526.000 Ergebnisse ${ }^{(51)}$. Das Konzept, Ironie zu nutzen, die breite Aufmerksamkeit generiert und es ermöglicht, eine Botschaft an möglichst viele Rezipienten zu senden, ist in diesem Fall beispielhaft dargelegt.

\section{Der Metallmix}

Ein weiterer Fall dieser positiven wirkenden Ironie, die von Beginn an beabsichtigt war, stellt der Wahlwerbespot der größten deutschen Einzelgewerkschaft, der IG Metall, dar. Dieses verbreitete sich wie ein Lauffeuer über die sozialen Netzwerke, erreichte schnell die Marke der 800.000 Views ${ }^{(52)}$. „Das Rezept ist einfach: Lustige Pannenvideos und Nachrichtenschnipsel werden zusammengemixt mit bekannten Filmszenen und dem Nachmittagsprogramm der Privatsender. Der Schnitt ist schnell, die

47 Vgl. Guido Herzog, „Ideenkopierer - Piraten in Niedersachsen starten in den Wahlkampf“, Flaschenpost, http://flaschenpost.piratenpartei.de/2012/11/23/ideenkopierer-piraten-in-niedersachsenstarten-in-den-wahlkampf/ (eingesehen 13.11.2013).

48 Wolfgang Kuhnle, „Die Ideenkopierer aus Niedersachsen“, Hochschule der Medien, 2012, http:// www.hdm-stuttgart.de/view_news?ident=news20121205180552 (eingesehen 13.11.2013).

49 Piratenpartei, „Warum häng ich hier eigentlich, ihr geht ja eh nicht wählen“, Piratenwiki, 2012, http:// wiki.piratenpartei.de/Datei:Piraten-AGH-Wahl-Warum-h\%C3\%A4ng-ich-hier-eigentlich.jpg (eingesehen 13.11.2013).

50 Vgl. Alexander Hensel, „Das Milieu der Piraten: Die Erben der Internetkultur“, in: Christoph BIEBer, Claus Leggewie (Hg.), Unter Piraten. Erkundungen in einer neuen politischen Arena, Bielefeld, transcript, 2012, S. 41-51; Stephan Klecha, Alexander Hensel, Zwischen digitalem Aufbruch und analogem Absturz: Die Piratenpartei, Opladen/Berlin/Toronto, Verlag Barbara Budrich, 2013, S. 25-33, bzw. S. 104-111ff.

51 (Stand: 12.11.2013).

52 Dogan Michael Ulosoy, „Wahlspot wird zum Hit aufYoutube“, Kölner Stadtanzeiger, 2013, http://www. ksta.de/bundestagswahl-2013/ig-metall-wahlspot-wird-zum-hit-auf-youtube,23500486,24225870. html (eingesehen 26.10.2013). 
Musik dramatisch, der Unterton ironisch bis bitterböse“(53) ${ }^{\text {. Das Video }}{ }^{(54)}$ ist ein Remix verschiedenster TV-Ausschnitte und versucht, der in der Endphase des Wahlkampfs verbreiteten Einschätzung, dass die Wahl längst entschieden sei, entgegenzuwirken. Bachl unterscheidet drei Kriterien, nach denen ein Video auf Erfolg überprüft werden könnte: Die Anzahl der Aufrufe, die Anzahl der Kommentare sowie die Unterscheidung zwischen positiven und negativen Äußerungen und schließlich die positiven Bewertungen ${ }^{(55)}$. Dennoch lässt die Anzahl der Views bis zur Entfernung des Videos vermuten, dass dies einer der erfolgreichsten Spots im gesamten Wahlkampf war, dessen Verbreitung hauptsächlich über die sozialen Netzwerke geschehen sein dürfte. „Der ,Metallmix“ trifft einfach nur den Ton des YouTube-Zeitalters“(56). Der Clip verfügt über alle Voraussetzungen, eine Filterblase zu durchbrechen. Zunächst einmal ist er ideologisch relativ indifferent. Politische Positionierungen im Video selbst werden zugunsten der Botschaft, wählen zu gehen, zurückgestellt. Der Ton ist streng ironisch gehalten. Das Bild des feststehenden Wahlausgangs wird durch viele Bilder, in denen sicher geglaubte Siege, für geklärt gehaltene Situationen ins Gegenteil sich verkehren, wirksam dekonstruiert und demaskiert.

\section{Fazit}

Abgestandener „alter Politik-Wein in neuen Kommunikations-Schläuchen“(57) - bei politischer Internetkommunikation ist dies sicherlich oft genug der Fall. Wenn Politik online geht, darf dieser Schritt aber nicht nur als partielle Ergänzung zur analogen politischen Kommunikation verstanden werden, sondern sollte mit dem Anspruch auf prozessuale Inklusion praktiziert werden. Essentiell dafür ist das Verständnis der Funktionslogik und der strukturellen Gegebenheiten des WorldWideWebs, die sich teils kongruent, teils divergent zu denen der analogen Welt verhalten. Das digitale Versprechen der großen Reichweiten beispielsweise stellt dabei eines der populärsten Luftschlösser dar. Denn die Ansprache potentieller Wähler oder gar der Nichtwähler gestaltet sich im Netz mindestens genauso komplex wie in der analogen Welt. Aufgrund von Filterblasen und interessengeleitetem, von Algorithmen beeinflussten Surfverhalten kann in den Social-Media-Communitys nicht die natürliche Heterogenität eines kleinstädtischen Marktplatzes erwartet werden. Über der digitalen politischen Kommunikation in Social-Media-Communitys schwebt daher, stärker noch als über den Wochenend-Wahlständen, das Damoklesschwert der Selbstreferentialität. Um dieser Gefahr entgegenzuwirken, um politische Statements über die Grenzen der eigenen Klientel hinaus zu verbreiten, bedarf eines strategischen Vorgehens. Vollzieht eine politische Botschaft den Weg aus ihrem Binnen- in den Außenkommunikationsraum, so erhöht sich die digitale Aufmerksamkeit. Ziel ist es dabei, unbeteiligte User

53 Timo BrüCKen, „Geht doch!“, stern.de, http://www.stern.de/politik/linsengericht/wahlspot-der-igmetall-geht-doch-2055179.html (eingesehen 26.10.2013).

54 Eine kommentierte Version des Videos befindet sich z.B. eingebettet auf stern.de im Artikel von T. BRÜCKEN, „Geht doch!“, ebd.

55 Vgl. M. BACHL, „Erfolgsfaktoren politischer YouTube-Videos“ (Anm. 15), S. 59f.

56 T. BRÜCKeN, „Geht doch!“ (Anm. 53).

57 Vgl. Leggewie/Bieber, „Interaktive Demokratie“ (Anm. 1), S. 37. 
zunächst überhaupt zu erreichen, diese zudem dazu zu animieren, die Botschaften unter den eigenen Freunden und Verwandten weiter zu verbreiten, sie am Ende gar als Wähler zu gewinnen. Um diesen Prozess jedoch zu initiieren, bedarf es eines Kommunikationsmodus, der politische Botschaften für eine breite Menge ansprechend verpackt. Wie bereits ausgeführt, werden von Usern insbesondere die Inhalte verbreitet, die grundlegend unterhaltenden und amüsierenden Charakter aufweisen. Nutzerinnen und Nutzer scheinen bevorzugt humorvolle Inhalte zu goutieren. Im Internet reüssiert, was witzig, ulkig oder ironisch ist.

Mit Blick auf die Verbreitung politischer Inhalte gestaltet sich dabei nicht die Erfassung von absoluten Klicks und Zugriffszahlen als interessant, sondern das zu Grunde liegende Prinzip der ironischen Durchbrechung von kommunikativen Grenzen. Anhand der aufgeführten Fallbeispiele konnten zwei Typen ironischer Wirkungsweise nachgezeichnet werden: Erstens die verkehrte Ironie, deren ursprünglich ernst gemeinter Inhalt erst seitens der Rezipienten einer Ironisierung unterliegt und so die Authentizität des Senders unterminiert. Wie ein unerwarteter Bumerang trifft seine negative Umdeutung den Sender selbst. Die eigentliche Botschaft tritt hinter die humoreske Reflektion zurück. Die Aufmerksamkeit der Netzgemeinde verlagert sich von der eigentlichen Botschaft fort zu dem widersprüchlichen Aspekt, der den Anknüpfungsmoment für die Ironie bildet. Der zweite Typ umfasst die genuine Ironie. Dieser Modus der Kommunikation basiert darauf, dass die eigentliche Botschaft gleich zu Beginn hinter, der von dem Sender bewusst genutzten Ironie, zurücktritt, um das Bewusstsein für die Kontingenz zu schaffen. Die Rezipienten nehmen zuerst die ironische Widersprüchlichkeit auf, um dann selbst-reflektiert die eigentliche Botschaft zu erkennen. Da der ironische Inhalt bewusster Teil der politischen Botschaft ist und im Anschluss von den Rezipienten nicht verfremdet, sondern in der ursprünglichen Art und Weise weiter getragen oder popularisiert wird, bleibt der Sender in seiner Authentizität nicht nur unberührt - die Authentizität vermittelt sich sogar explizit über den ironischen Modus als solchen. Die Ironie schlägt hier Brücken zwischen diversen Interessen- und homogenen Kleinstgruppen.

All das lässt sich für den abgelaufenen Bundestagswahlkampf geradezu musterhaft zeigen. Soweit die Parteizentralen ihre Budgets für Onlineaktivitäten eingesetzt haben, handelte es sich um vergleichsweise konventionelle Angebote im Gewand interaktiver Dienste. Anzeigen bei den Onlineausgaben der großen Zeitungen substituieren das Budget für Zeitungsanzeigen. Kontaktformulare und Banner auf Internetseiten ersetzen die klassische Wurfsendung oder das Angebot einer postalischen Kontaktaufnahme des Wählers zu den Parteien. Wirklich spezifisch wird politische Kommunikation im Internet immer erst dann, wenn sie die humoristisch-ironische Stufe überschreitet. Das mag in einzelnen Fällen kalkulierbar sein, in vielen Fällen ist es das aber nicht. 


\section{Zusammenfassung}

Mittels einer breiten digitalen Wahlkampfkommunikation versuchen Parteien ihre politischen Themen und Positionen, über die Ansprache der eigenen Parteiklientel hinaus, an generell unentschlossene oder bereits sympathisierende Wähler heranzutragen. Diese strategische Absicht kollidiert jedoch mit den Funktionsweisen des Internets und der Social Media Communitys. Denn dort bilden sich Gruppen vorzugsweise anhand ähnlicher Interessen oder politisch übereinstimmender Meinungen. Daraus resultiert die verstärkte Bildung von Blasen der Selbstreferentialität. Eine solche Filter Bubble lässt sich kommunikativ nur äußerst schwierig überwinden, die Ansprache neuer Wählergruppen wird erschwert. Bei der Wahlkampfkommunikation über parteieigene Kanäle wird somit tendenziell nur die eigene Klientel erreicht. In diesem Artikel wird ausgeführt, wie die Verwendung von Ironie erlaubt, wahlkampfpolitische Inhalte brückenhaft zu transportieren. Anhand beispielhafter Fälle werden zwei Typen der ironischen Wirkungsweise nachgezeichnet: die verkehrte Ironie sowie die genuine Ironie.

\section{Abstract}

To reach voters beyond their generic clientele who are generally undecided or already sympathetic political parties use broad digital election campaigns for communicating their political issues and positions. This strategy, however, collides with the way the internet in general and social media communities in particular work. Here, groups preferably form on the basis of similar interests or opinions that coincide politically. As a result, bubbles of "self-referentiality" build increasingly. It is very difficult to overcome such filterbubbles in ways of communication so that addressing new voters is rather impeded. Therefore, election campaign communication that only uses party specific channels will mainly reach the own clientele. This article illustrates how implementing irony as a communicative bridge can transport political issues. Derived from exemplary cases two kinds of irony are described here: an inverted irony as well as a genuine irony.

\section{Résumé}

Lors des campagnes électorales, les partis politiques essayent de diffuser via Internet au plus grand nombre leurs valeurs et leurs positions. Ils ciblent non seulement leurs adhérents mais aussi des électeurs indécis ou des sympathisants. Cette stratégie de partis est en contradiction avec le mode de fonctionnement habituel d'Internet et des réseaux sociaux. Les communautés s'y créent autour d'intérêts similaires ou autour d'opinions politiques communes. Il en résulte la formation de "Filterbubbles »-des communautés numériques fermées sur elles-mêmes, qui se caractérisent par une logique de fonctionnement hermétique à leur environnement. Il est extrêmement difficile de s'adresser à des nouveaux électeurs en dehors de ces "filterbubbles" à cause des barrières communicatives propres à ce système fermé. La conséquence est que les partis politiques qui utilisent leurs propres canaux de communication n'atteignent que leur public sympathisant. Cet article explique comment l'emploi de l'ironie permet de transmettre les programmes lors des campagnes électorales. Le mode d'action de deux types d'ironie est détaillé à travers des exemples précis : l'ironie travestie et l'ironie originale. 